\title{
Neuromuscular Electrical Stimulation: A Promising Modality of Exercise Training in Cardiovascular Rehabilitation Phase I?
}

\author{
Sergio Chermont ${ }^{1,2}$ and Mônica Quintão ${ }^{1,3}$ \\ Universidade Federal Fluminense, ${ }^{1}$ Niterói, RJ - Brazil \\ Hospital Santa Martha, ${ }^{2}$ Rio de Janeiro, RJ - Brazil \\ Instituto Nacional de Câncer, ${ }^{3}$ Rio de Janeiro, $R J$ - Brazil
}

Editorial related to the article: Neuromuscular Electrical Stimulation on Hemodynamic and Respiratory Response in Patients Submitted to Cardiac Surgery: Pilot Randomized Clinical Trial

There are currently few indications for the use of neuromuscular electrical stimulation (NMES) in cardiac patients that are based on systematic reviews or even meta-analyses from the perspective of evidence-based practice. Therefore, the therapeutic potential of NMES is probably still poorly explored. Previous studies have shown benefits of NMES in patients with stable chronic heart failure (CHF). ${ }^{1}$ However, we wonder whether the application of this technique in cardiac patients has been extensively explored. We are certainly dealing with a new therapeutic possibility so far rarely recommended and employed in patients with cardiovascular diseases, especially in intrahospital cardiovascular rehabilitation. In this scenario, what could be added to the current knowledge and its main indications?

In the absence of well-defined protocols, feasibility and safety studies may be considered as a starting point for further studies and for paving the way clinical trials and to determine whether or not the conduct is favorable for a patient's specific clinical condition. So, what would be the role of NMES in heart surgery?

Cerqueira et al., ${ }^{2}$ used NMES in patients undergoing cardiac surgery in the immediate postoperative period. This study evaluated the effects of NMES on systolic blood pressure, diastolic blood pressure and heart rate (HR) and peripheral oxygen saturation, which can be considered as independent predictive variables with

\section{Keywords}

Heart failure; Thoracic Surgery; Transcutaneous Electric Nerve Stimulation; Cardiac Rehabilitation. a negative impact on adverse events in the immediate postoperative period of cardiac surgery. ${ }^{2}$

The authors in this study demonstrated that in the first $23.13 \pm 5.24$ hours after cardiac surgery there were no abnormalities in hemodynamic and respiratory parameters between patients who underwent NMES and their respective control group. ${ }^{2}$ By investigating the feasibility and safety of the use of this technique in patients, Cerqueira et al. ${ }^{2}$ reported that, in their sample, NMES did not determine significant abnormal findings that could result in risk to patients in the immediate postoperative period. ${ }^{2}$

NMES can be considered a promising new modality of exercise training in cardiovascular rehabilitation (CVR). In fact, the use of this technique in patients with heart failure (NYHA III/IV) and reduced functional capacity led to improved exercise tolerance in those patients who could not perform conventional exercises..$^{3-5}$ According to meta-analyses on the use of NMES, Cerqueira et al. used this technique as intensely as in other studies that produced benefits in patients with $\mathrm{HF} .{ }^{1,5}$

During phase I of CVR, as in the case of patients in the postoperative period of cardiac surgery, conventional exercise is recommended as the first choice, especially in individuals who have the ability to do so. ${ }^{6}$ Patients in the postoperative period of cardiac surgery often present loss of cardiopulmonary capacity. A recent study evaluated four different CVR protocols in 40 patients in the postoperative period of cardiac surgery. Protocols that included early ambulation and exercise of the four segments decreased in-hospital functional losses, improved recovery and walking distance. ${ }^{7}$ This type of 
protocol, even when done briefly, is safe and effective for these patients. ${ }^{8}$

However, evidence such as this one does not make it impossible for NMES to be used as an adjuvant treatment or in patients with no functional capacity to perform dynamic exercises. Therefore, the indication and prescription of the modality to be performed should be analytical and precise, considering that most of the progress in research studies showing the benefits of NMES converge to the scenario of patients with HF who cannot perform conventional dynamic exercises. ${ }^{1,5}$

NMES does not yet have a well-defined role in the postoperative period of cardiac surgery, and little is known about whether it would play an important role in early physiological adaptations in an intrahospital CVR protocol.

The intensity of acute physiological responses to exercise involves increased $\mathrm{HR}$, ventilation, catecholamine release, and autonomic responses, which reflect an individual's ability to undergo repetitive and regular adjustments that determine physiological adaptations which promote increased performance and exercise capacity. ${ }^{9}$

Cerqueira et al., ${ }^{2}$ found that the absence of hemodynamic abnormalities could be an indication of a safety parameter. However, in the scenario of phase I CVR, in order to use NMES as an adjuvant technique, hemodynamic abnormalities must exist to ensure progressive adaptations to exertion. ${ }^{9}$

Otherwise, the investigation of restricted hemodynamic parameters may not reflect the true extent of response to NMES. It is important to measure other hemodynamic parameters both for patient safety and to infer physiological adaptations, such as systolic volume, cardiac output and peripheral vascular resistance response, etc., which could provide guidance in the immediate postoperative period of cardiac surgery, especially in the presence of vasopressor use. ${ }^{3}$

Unlike Cerqueira et al., ${ }^{2}$ a recent study demonstrated changes in both systolic volume, cardiac output and systemic vascular resistance from using NMES in patients with HF and reduced ejection fraction. ${ }^{10}$

The design and methodology applied in the study by Cerqueira et al., ${ }^{2}$ were careful and sufficient to confirm the hypothesis, but measurements of other more complex hemodynamic and autonomic responses may actually ensure feasibility and safety and provide more information about the mechanism of benefit and usefulness of this technique as an adjuvant resource for intrahospital CVR. Although the intensity of NMES used is compatible with most studies, other intensities can also be tested and compared to measure the magnitude of their responses. Further studies are needed to pave the way for the implementation and corroboration of new protocols with NMES.

\section{References}

1. Gomes Neto M, Oliveira FA, Reis HF, de Sousa Rodrigues- E Jr, Bittencourt HS, Oliveira Carvalho V. Effects of Neuromuscular Electrical Stimulation on Physiologic and Functional Measurements in Patients With Heart Failure: A Systematic Review With Meta-Analysis. J Cardiopulm Rehabil Prev. 2016;36(3):157-66.

2. Cerqueira TC, Neto ML, Carvalho AJ, Oliveira GU, Filho AA, Carvalho VO, et al. Neuromuscular Electrical Stimulation on Hemodynamic and Respiratory Response in Patients Submitted to Cardiac Surgery: Pilot Randomized Clinical Trial. Int J Cardiovasc Sci. 2019;32(5):483-489.

3. Forestieri P, Bolzan DW, Santos VB, Moreira RSL, de Almeida DR, Trimer $\mathrm{R}$, et al. Neuromuscular electrical stimulation improves exercise tolerance in patients with advanced heart failure on continuous intravenous inotropic support use-randomized controlled trial. Clin Rehabil. 2018 Jan;32(1):66-74.

4. Hainaut K, Duchateau J. Neuromuscular electrical stimulation and voluntary exercise. Sports Med. 1992;14(2):100-13.

5. Sbruzzi G, Ribeiro RA, Schaan BD, Signori LU, Silva AM, Irigoyen MC, et al. Functional electrical stimulation in the treatment of patients with chronic heart failure: a meta-analysis of randomized controlled trials. Eur J Cardiovasc Prev Rehabil. 2010;17(3):254-260.

6. Herdy AH, López-Jiménez F, Terzic CP, Milani M, Stein R, Carvalho T, et al. Sociedade Brasileira de Cardiologia. Diretriz Sul-Americana de prevenção e reabilitação cardiovascular. Arq Bras Cardiol. 2014;103(2):1-31.

7. Zanini M, Nery R, Lima J, Buhler R, Silveira R, Stein R. effects of different rehabilitation protocols in inpatient cardiac rehabilitation after coronary artery bypass graft surgery: a randomized clinical trial. J Cardiopulm Rehabil Prev. 2019 Jul . doi: 10.1097/HCR.0000000000000431. [Epub ahead of print]

8. Spiroski D, Andjić M, Stojanović O, Lazović M, Dikić AD, Ostojić M, et al. Very short/short-term benefit of inpatient / outpatient cardiac rehabilitation programs after coronary artery bypass grafting surgery. Clin Cardiol. 2017;40(5):281-6.

9. Nobrega AC. The subacute effects of exercise: concept, characteristics, and clinical implications. Exerc Sport Sci Rev. 2005;33(2):84-7.

10. Dias DW, Marchese LD, Muradas MC, Mascarenhas C, Ferreira BA, Branco WD, et al. Efeito agudo de uma sessão de eletroestimulação neuromuscular sobre as variáveis hemodinâmicas em pacientes com insuficiência cardíaca. Rev Bras Cardiol. 2014;27(Suppl A):1-5. 